\title{
Qualität der Lehre - wesentliches Erfordernis einer effizienten Fachhochschul-Ausbildung
}

\author{
Prof. Dipl.-Ing. Alfred Hentschel
}

Den Ausgangspunkt für eine Diskussion zum genannten Thema bilden Studentenbefragungen zur Dozentenbeurteilung und von Studienanfängern bezüglich ihrer Erwartungen zum Studium an der Technischen Fachhochschule Wildau [1], [2].

Der Zeitpunkt der Diskussion zu den ausgewählten Schwerpunkten, die jeweils studentische Anliegen betreffen, erscheint am Ende der Gründungsphase der Hochschule geeignet, um aus der Sicht des Lehrenden mit den in den 4 Gründungsjahren gesammelten Erfahrungen einmal auf diese Anliegen einzugehen.

Dies soll letztlich auch mit dem Ziel getan werden, die Attraktivität des Studienstandortes Wildau zu untersuchen.

Der Rahmen dieser Betrachtungen ist wegen der Zugehörigkeit ausschließlich auf den Fachbereich Maschinenbau begrenzt.

Der Verfasser hat einerseits mit dem Lehrfach „Fertigungsverfahren" (1.-3. Semester) Erfahrungen mit Studienanfängern sammeln können und andererseits mit dem Lehrfach „Arbeitsvorbereitung“ (4.-7. Semester) Einblick in die Probleme der „fortgeschrittenen" Studenten erhalten.

\section{Lehren muß gelernt werden}

Wie lernt ein Hochschullehrer das Lehren? Auf diese einfache, aber wie die Studentenbefragungen letztlich zeigen, höchst wichtige Frage weiß man heute keine rechte Antwort zu geben.

Zwar wird die wissenschaftliche Kompetenz eines Nachwuchswissenschaftlers über die Qualitätskriterien Promotion, Habilitation und Zahl der Publikationen gründlich abgeklopft.

Bei der Fähigkeit, das Wissen auch an Studenten zu vermitteln, vertraut die Berufungskommission z. B. auf die Probevorlesung oder schlicht auf das intuitive pädagogische Geschick des akademischen Nachwuchses. Oder es existiert die Hoffnung, der aufstrebende Wissenschaftler werde sich das notwendige didaktische Rüstzeug in den eigenen Lehrveranstaltungen mit der Zeit gewissermaßen (von) selbst aneignen.

Aber funktioniert dieses „learning by doing"? Leider nur zum Teil, wie auch die Studentenbefragungen an der TFH Wildau zeigen. Zwar gibt es unter den Professoren und Dozenten einige, die die Studierenden regelrecht in ihren Bann ziehen, aber eben auch solche, die in Sachen Vermittlungskompetenz eher schwächer eingeschätzt werden.

Wie sich das ändern läßt? Seit einiger Zeit gibt es durch- aus ernstzunehmende Ansätze zur Lehr-Verbesserung:

- etwa das Bestreben einiger Bundesländer, die Anforderungen der Berufung zum Professor nicht nur auf wissenschaftliches Können zu beziehen, sondern auch auf die bereits praktische erwiesene Lehrbefähigung des Kandidaten,

- oder die bundesweite, vom Ring Christlich-Demokratischer Studenten (RCDS) organisierte Fragebogenaktion „Prüf den Prof“, bei der Studierende die Qualität von Lehrveranstaltungen und die Betreuung durch die Hochschullehrer, aber auch die Rahmenbedingungen am Fachbereich beurteilen.

Derartige Hoschulratings erscheinen dann nützlich, wenn sie dem einzelnen Professor als Rückmeldung dienen, damit dieser z. B. seine Veranstaltungen überdenken und gegebenenfalls verbessern kann.

- Andere Ansätze haken bei dem Defizit ein, daß es in Deutschland - im Gegensatz zu Ländern wie den USA, Kanada oder Neuseeland - keinen rechten Ansporn zu didaktisch ansprechenden Vermittlungsleistungen gibt. Von dieser Diagnose des Bielefelder Hochschuldidaktikers Wolff-Dietrich Webler inspiriert richtete das baden-württembergische Wissenschaftsministerium im vergangenen Jahr $\mathrm{z}$. B. einen Landeslehrerpreis ein, der alljährlich an in der Lehre besonders ambitionierte Mitarbeiter der Hochschulen im Land vergeben wird.

Zu diesen Lösungsmöglichkeiten vertritt der Verfasser die Meinung, daß Preise allein keine grundlegende Verbesserung der Lehre bewirken können, ebensowenig wie ein umfangreiches Studium der Hochschuldidaktik. Vielmehr sollte der potentielle Bewerber für ein HochschulLehramt neben der wissenschaftlichen Kompetenz in seinem Fachgebiet Grundkenntnisse der Hochschuldidaktik besitzen und sich dann das spezifische didaktische Rüstzeug durch „learning by doing“ selbst aneignen.

Als Regulativ könnte in diesem Prožeß die direkte Befragung der Studierenden durch den Lehrenden dienen oder wegen größerer Objektivität die anonyme Dozentenbeurteilung.

\section{Prüfstand für Professoren und Dozenten}

Galt eine studentische oder gar amtliche Beurteilung der Lehrtätigkeit noch vor wenigen Jahren vielen „akademischen Halbgöttern" als schamlose Beeinträchtigung ihrer Lehrfreiheit, so erscheint diese Art der Leistungskontrolle den meisten inzwischen als geeignetes Instrument 
zur Optimierung des Ausbildungsangebotes im Dienstleistungsbetrieb Hochschule.

Sie setzt ihren ganzen Stolz darein, stärker als alle anderen wissenschaftlichen Einrichtungen Forschung und Lehre miteinander zu verbinden. Gerade wegen der Ausbildung der jungen Generation verstehn sie sich als die „Mutterwesen der Wissenschaft“, so Hans-Uwe Eichsen, Präsident der Hochschulrektorenkonferenz.

In diesem Rahmen haben natürlich Fachhochschulen ihre spezifischen Aufgaben, auf die in den folgenden Punkten noch näher einzugehen ist.

Zudem hat eine neugegründete $\mathrm{FH}$ in den neuen Bundesländern zur Erlangung der von ihr gewünschten Attraktivität noch vordringlicher die Pflicht, solche Regularien wie die Studentenbefragung zu nutzen.

Ihr Signalwert hat doch mehrfache Wirkung:

- zum einen als Hinweis darauf, daß man Lehre wichtig nimmt,

- zum anderen bewirkt sie „automatisch“, daß man sich Gedanken über die Kriterien für gute Lehre macht ( $\mathrm{z}$. B. in Fachbereichsvollversammlungen, wo bei solch wichtigen Themen auch Studentenvertreter zugegen sein sollten),

- nicht zuletzt setzen solche Befragungen zugegebenermaßen bei der Eitelkeit der Professoren und Dozenten an.

Es gibt auch aus der Sicht des Verfassers nur Vorbehalte gegen eine förmliche Bewertung oder „Evaluation“ der Lehre in Bezug auf die Urteilskriterien.

Die Ziele und Methoden müssen von Studenten, Professoren, Prüfungsämtern und zuständigen Ministerien gemeinsam entwickelt werden, also im Interessenkompromiß.

Sonst hätten die Ergebnisse zwar ein mehr oder weniger aufsehenerregendes Nachspiel in der Öffentlichkeit, aber kaum Folgen im Hochschulalltag.

Außerdem muß beachtet werden, wie weit die Ergebnisse bei verschiedenen Hochschullehrern miteinander vergleichbar sind oder daß bei gleichermaßen notwendigen Lehrstoffen von vornherein mit unterschiedlichem Widerhall zu rechnen ist.

Und nicht zuletzt müssen die Befragungen selbstverständlich repräsentativ sein, was bei der Dozentenbeurteilung der TFH Wildau [2] durch $26 \%$-ige Studententeilnahme etwas fraglich erscheint.

Trotzdem soll im folgenden auf die dort aufgeworfenen Fragen oder Kritikpunkte eingegangen werden.

\begin{tabular}{|c|c|c|c|c|c|c|}
\hline \multicolumn{7}{|c|}{$\begin{array}{l}\text { Warum haben Sie das Fachhochschulstudium de } \\
\text { Universität vorgezogen? (Angaben in Prozent) }\end{array}$} \\
\hline & NBL & $\mathrm{ABL}$ & männl. & weibl. & WiWi. & Ing. \\
\hline $\begin{array}{l}\text { praxisverbundene } \\
\text { Ausbildung }\end{array}$ & 82 & 92 & 85 & 79 & 83 & 80 \\
\hline berufsnähere Ausbildung & 78 & 67 & 73 & 83 & 70 & 84 \\
\hline schnellerer Abschluß & 62 & 67 & 63 & 67 & 70 & 52 \\
\hline bessere Betreuung & 62 & 67 & 65 & 58 & 68 & 52 \\
\hline bessere Berufschancen & 54 & 50 & 55 & 50 & 50 & 56 \\
\hline $\begin{array}{l}\text { Ich habe mich in 1. Wahl } \\
\text { für eine Fachhochschul- } \\
\text { ausbildung entschieden. }\end{array}$ & 74 & 58 & 70 & 75 & 73 & 68 \\
\hline
\end{tabular}

Tabelle: Entscheidung für den Hochschultyp an Hand berufsorientierter Studienmotive (Auszug aus [1])

\section{Praxisverbundene und berufsnähere Ausbildung}

Diese Zielstellungen sind zwar weitgehend durch die ministeriell bestätigten Lehrprogramme vorgeschrieben, aber es bleibt für die Hochschule und den Hochschullehrer noch genügend Spielraum zu deren Umsetzung. Dies geht bis zu Fragen der Darbietungsform des Lehrstoffes, wie sie in [2] gestellt wurden, z. B.:

- ist die Lehrveranstaltung klar strukturiert,

- ist das Interesse für den Lehrstoff angeregt worden,

- effektiver Einsatz von Unterrichtshilfen,

- wird das Vorwisssen des Studenten berücksichtigt, usw.

In den Lehrfächern „Fertigungsverfahren“ und „Arbeitsvorbereitung" ist der Lehrinhalt klar strukturiert und orientiert sich vor allem am künftigen Tätigkeitsfeld des Absolventen. Der Lehrstoff selbst wird nicht einfach im üblichen Vorlesungsbetrieb, sondern, wo immer sich es anbietet, gemeinsam mit den Studenten erarbeitet und bezieht sich auf praxisnahe Fallbeispiele oder Problemstellungen. So fachübergreifend wie diese muß selbstverständlich vorher eine Abstimmung zwischen Lehrfächern realisiert sein. Hierzu besitzt eine kleine Fachhochschule bei Beachtung der sonstigen Forderung, z. B. nach Freiheit der Lehre, gute Voraussetzungen.

Die vom Verfasser vertretenen Lehrfächer sind eng verzahnt mit:

- allen Gruppen (Teilgebieten) der Fertigungsverfahren,

- Werkstofftechnik,

- Fertigungsmeßtechnik/Qualitätssicherung,

- Fertigungssysteme,

- Werkzeugmaschinen,

- Fabrik- und Materialflußplanung,

- Rechnerintegrierter Betrieb.

An der TFH Wildau wurde zudem immer sehr großer Wert auf eine umfangreiche Laborausbildung gelegt. Im Grundstudium beträgt z. B. der Anteil der Laborstunden neben den Vorlesungen und Übungen $21 \%$. Die Fertigungsverfahren besitzen gut ausgestattete Labors für die Teilgebiete Urformen, Umformen, Trennen, Fügen, d. h. die Laborausbildung reicht hier von Einformübungen, die in besonderem Maße im 1. Semester das räumliche Vorstellungsvermögen des späteren Konstrukteurs oder Fertigungsingenieurs entwickeln sollen, bis hin zu Verfahrensuibungen zur spezielleren Teilefertigung, z. B. der Verzahnung.

Im Hauptstudium Fertigung beträgt dẹr Laboranteil 31\% der Gesamtstunden und im Lehrfach „Arbeitsvorbereitung" speziell $40 \%$. Hier reicht die Übungspalette von elementaren Aufgaben, wie:

- Bestimmung der Arbeitsvorgangsfolge,

- Zeit- und Kostenberechnungen,

- optimale Rohteilauswahl,

- Losgrößenermittlung,

- Untersuchungen zur Mehrstellenarbeit usw.

bis hin zur Programmierung von rechnergeführten Fertigungsmitteln (manuelle Programmierung von CNC-Maschinen) sowie mit dem Grafiksystem „QUANTUM“ die Programmierung von Koordinatenmeßtechnik. 
Sowohl für die Fertigungsverfahren als auch die Arbeitsvorbereitung wird seit kurzem in der Laborausbildung das Softwarepakete „COROPLAN“ zur

- Auswahl spannender Werkzeuge für Drehen/Fräsen,

- Bestimmung der Arbeitsvorgangsfolge,

- Ermittlung von Schnittdaten,

- Bestimmung von Zerspanungszeiten und -kosten eingesetzt, um die Studenten mit Problemen einer wirtschaftlichen Fertigung zu konfrontieren.

Alle Aufgabenstellungen im Labor „Arbeitsvorbereitung“ enden praktisch mit der Simulation eines konkreten Fertigunssablaufes in einem fiktiven Klein- bzw. mittelständischen Unternehmen des Maschinenbaus.

Diese Methodik wird im AV-Labor bereits seit mehreren Jahren angewandt, lediglich das Fertigungsprogramm und die -einrichtungen werden in gewissen Zeitabständen verändert oder aktualisiert.

Nach neuerlicher Kontaktaufnahmen zu Unternehmen der Region ist beabsichtigt, auch konkrete Praxisaufgaben in Form von Studienarbeiten in beiden Labors durch Studenten lösen zu lassen.

Zudem werden fest eingeplante Exkursionen in ausgewählte Unternehmen (z. B. Wildauer Kurbelwelle GmbH, PLM-Dosenfabrik Berlin-Zehlendorf) zur Unterstuitzung der Lehre genutzt.

All diese Beispiele sind immanenter Bestandteil der Lehrprogramme, d. h. die berechtigten Forderungen nach Praxisverbundenheit und Berufsnähe in der Ausbildung werden jedem Studenten im Rahmen seiner Regelstudienzeit erfuillt.

Dies ist in der Hochschullandschaft sicherlich nicht überall realisierbar, aber die abgestimmte Vorgehensweise in der Lehre und damit die Flexibilität ermöglicht eben eine kleinere $\mathrm{FH}$.

\section{Bessere Berufschancen durch bessere Betreuung der Studenten}

Eine berufsrelevante Ausbildung des Studenten darf sich selbstverständlich nicht nur auf die Lehre beschränken, sondern muß auch an einer FH genauso die Forschung mit einbeziehen.

Auf diesen Aspekt wird hier nicht näher eingegangen, sondern bleibt einer späteren Erörterung vorbehalten. Es ist so, daß bei der Bewerbung des Absolventen nicht nur die Zeugnisnoten oder das Prädikat der Abschlußprüfung eine Rolle spielen, sondern zumindest gleichberechtigt wird auch seine Betätigung in den anderen Studienabschnitten, z. B. im Praxissemester oder beim Diplomverfahren, hinterfragt werden.

Hier muß im Hinblick auf die Vermittelbarkeit in eine zukünftige ingenieurmäßige Tätigkeit auf anspruchsvolle Aufgabenstellungen mit hohem fachlichen Niveau Wert gelegt werden. Dies trifft wie gesagt auch auf das Praxissemester zu, denn es geht nicht an, dort lediglich einen Durchlauf im Unternehmen zu absolvieren.

Und schließlich hat die Kontaktaufnahme zu Unternehmen beispielsweise im Praxissemester ja noch andere Effekte:
- Bekanntwerden der FH,

- Studienbewerbung,

- Weiterführung von Aufgaben im Diplomsemester,

- Übernahme von Forschungsaufgaben usw.

Deshalb wurden gerade in den letzten beiden Jahren durch den Verfasser solche Kontaktaufnahmen getätigt und mit anspruchsvollen Aufgabenstellungen untersetzt:

INA-Werk Schaeffler AG, Herzogenaurach:

- Untersuchungen von Kühl- und Schmiermitteln,

- Veränderung der Schneidegeometrie zur optimalen Spanbildung bei der Bearbeitung auf MehrspindelDrehautomaten.

Nutzkraftwagen Ludwigsfelde GmbH (Betrieb der Mercedes Benz $A G)$ :

- Fertigungsablauforganisation,

- Instandhaltung.

Wildauer Kurbelwelle GmbH:

- Untersuchung der Fertigungsverfahren und Reengineering des Materialflusses in der Schmiede,

- Betriebsstättenplanung Kurbelwellen-Endbearbeitung,

- Entwicklung von Transportsystemen,

- Erweiterung der Technologiebausteine für ein CNCProgammiersystem,

- Kopplung Meßdatenerfassung und CNC-Programm,

- Verfahrens- oder Fertigungsmitteluntersuchungen beim Verrunden von Schmierlochbohrungen,

- Optimierung der Vorformung beim Gesenkschmieden,

- Optimierung des Dreh- und Radienfräsens usw.

Ein anderes Feld zur besseren Vermittelbarkeit des Absolventen wurde ebenfalls erkannt und zielgerichtet ausgebaut. Es geht um die zusätzliche Vergabe von Qualifikationsnachweisen zum FH-Diplom.

Durch die Kontaktaufnahme zum REFA-Verband BerlinBrandenburg wurde ermöglicht, daß die Absolventen den REFA-Grundschein erlangen können, indem ihnen $2 / 3$ des sonstigen Umfangs dadurch anerkannt werden, daß die Lehrinhalte des Faches „Arbeitsvorbereitung“ in der FH-Ausbildung ohne Änderung diesem Anspruch gerecht werden und lediglich $1 / 3$ des Umfangs in einem Wochenlehrgang mit erheblich vermindertem persönlichen Kostenaufwand noch zu belegen sind.

Ähnliches wird im Teilgebiet „Fügen“ realisiert. Dies wird zwar nicht vom Verfasser betreut, gehört aber lehrseitig bekanntermaßen zu den Fertigungsverfahren und ist ebenfalls ein gutes Beispiel in diesem Rahmen.

Der Absolvent kann die Zusatzqualifikation „Schweißfachingenieur" durch die Schweißtechnische Lehr- und Versuchsanstalt (SLV) erlangen, indem die TFH Wildau im Lehrfach „Fügen“ ca. 110 Std. für den Teil I erbringt und der andere ca. 50\%-ige Stundenumfang für den Teil II fakultativ durch die SLV selbst erbracht wird.

All diese Beispiele zeigen, daß die neugegründete TFH Wildau ein attraktives Lehrangebot installiert hat. Selbstverständlich ist dies auch in Bezug auf die Zusammenarbeit mit Unternehmen der Region oder die Labor- 
ausbildung noch ausbaufähig. Die Kapazitäten dazu existieren und müßten in den Studienplänen weitere Berücksichtigung finden.

Schließlich tragen diese Studienbedingungen und nicht zuletzt die darüber hinausgehenden guten sonstigen Bedingungen an der TFH (Wohnheim, bevorstehende flächenmäßige Erweiterung der Hochschule) zur Werbung und damit Attraktivität des Studienstandortes bei. Und wenn dann noch in den Werbebroschüren Berliner Studienberatungsstellen - so die selbstgemachten Erfahrungen bereits immatrikulierter Studenten - der Studienstandort Wildau geografisch nicht in der „Nähe von Dresden“, sondern in unmittelbarer Nähe zur Stadtgrenze Berlins zu finden wäre, dann hätte sicherlich die Werbung größere Effizienz.

\section{Quellen}

[1] Projektgruppe Hochschulforschung Berlin-Karlshorst: Befragung von Studienanfängern im Wintersemester 93/94 an der Technischen Fachhochschule Wildau, Berlin, März 1994

[2] Studentenrat der TFH Wildau: Dozentenbeurteilung im Sommersemester 1994 an der TFH Wildau, Wildau, 07. Nov. 1994

[3] Hochschule und Bildung - Hochschuldidaktik, in: „Rheinischer Merkur“, Nr. 49, 09. Dez. 1994

\section{Verfasser}

Prof. Dipl--Ing. Alfred Hentschel

Technische Fachhoschule Wildau

Fachbereich Maschinenbau

Tel. (03375) 507-117 